\title{
A DIRECT PROOF THAT THE RC-INTEGRAL IS EQUIVALENT TO THE D*-INTEGRAL
}

\author{
YÔTO KUBOTA \\ ABSTRACT. We give a direct and short proof that the Riemann complete integral \\ defined by $R$. Henstock is equivalent to the special Denjoy integral.
}

1. Introduction. It is well known that the Riemann complete integral (RC-integral) defined by $R$. Henstock in [4] is equivalent to the special Denjoy integral ( $\mathrm{D}^{*}$-integral). This fact is proved by the following procedure. The RC-integral is equivalent to the variational integral [3] which is also equivalent to the Ward integral [2]. The Ward integral is equivalent to the Perron integral [6] which is, in turn, identical with the $\mathrm{D}^{*}$-integral by the Theorem of Hake-Alexandroff-Looman [5].

The object of this paper is to give a direct and short proof of this equivalence. For fundamental terminologies and notations on the RC- and $\mathrm{D}^{*}$-integrals, we refer to [3] and [5] respectively.

If $J=[\alpha, \beta]$, then $J^{0}$ is the open interval $(\alpha, \beta)$.

2. The Riemann complete integral. We begin by reproducing the definition of the RC-integral for completeness [4].

Let $f$ be a real-valued function defined on a compact interval $I_{0}=[a, b]$. Then $f$ is said to be RC-integrable on $[a, b]$ to the value $A=(R C) \int_{a}^{b} f$ if, for any $\varepsilon>0$, there exists a positive real-valued function $\delta(x)$ on $[a, b]$ such that whenever $a=x_{0}<x_{1}<\cdots<x_{n}=b$ and $x_{i-1} \leqslant \xi_{i} \leqslant x_{i}, \quad x_{i}-x_{i-1}<\delta\left(\xi_{i}\right) \quad(i=$ $1,2, \ldots, n)$, we have

$$
\left|\sum_{i=1}^{n} f\left(\xi_{i}\right)\left(x_{i}-x_{i-1}\right)-A\right|<\varepsilon .
$$

We state some important properties of the RC-integral which will be used in what follows.

A finite function $F$ is said to be $\mathrm{AC}^{*}$ on $E$, if $F$ is bounded on an interval containing $E$ and if to each $\varepsilon>0$ there corresponds a $\delta>0$ such that, for every finite sequence of nonoverlapping intervals $\left\{I_{k}\right\}$ whose end points belong to $E$, the inequality $\Sigma_{k}\left|I_{k}\right|<\delta$ implies $\Sigma_{k} O\left(F, I_{k}\right)<\varepsilon$, where $O\left(F, I_{k}\right)$ means the oscillation of $F$ on $I_{k}$. A function will be termed $\mathrm{ACG}^{*}$ on $E$, if the function is continuous on $E$ and if $E$ is expressible as the sum of a sequence of sets on each of which the function is $\mathrm{AC}^{*}$.

Received by the editors February 13, 1979.

AMS (MOS) subject classifications (1970). Primary 26A39.

Key words and phrases. Riemann complete integral, special Denjoy integral, regular interval. 
Property 1 ([3, Theorems 30.3, 32.1 AND 35.1]). If $f$ is RC-integrable on $I_{0}$ then its indefinite integral $F(x)$ is $\mathrm{ACG}^{*}$ on $I_{0}$ and $F^{\prime}(x)=f(x)$ a.e.

Property 2 ([1] OR [4]). If $f$ is L-integrable on $I_{0}$, then $f$ is also RC-integrable on $I_{0}$ and $(R C) \int_{I_{0}} f=(L) \int_{I_{0}} f$.

Property 3 ([3, TheOREM 46.1]). If $f$ is RC-integrable on every interval $[a, \beta]$ $(a<\beta<b)$ and if $\lim _{\beta \rightarrow b}(R C) \int_{a}^{\beta} f=A$, then $f$ is RC-integrable on $[a, b]$ and $(R C) \int_{a}^{b} f=A$.

Property 4 ([3, Theorem 48.1]). If $f$ is L-integrable on a closed set $E$ with end points $a, b$ and complementary intervals $\left\{\left(a_{n}, b_{n}\right)\right\}$, while $f$ is RC-integrable on each interval $I_{n}=\left[a_{n}, b_{n}\right]$, and if $\Sigma_{n} O\left(F_{n}, I_{n}\right)<\infty$, where $F_{n}$ is an indefinite integral of $f$ on $I_{n}$, then $f$ is RC-integrable on $[a, b]$ and

$$
(R C) \int_{a}^{b} f=(L) \int_{E} f+\sum_{n=1}^{\infty}(R C) \int_{I_{n}} f .
$$

\section{Proof of the equivalence.}

THEOREM 1. If $f$ is $R C$-integrable on $[a, b]$ then for any closed set $E \subset[a, b]$, there exists a closed interval $J$ with $J^{0} \cap E \neq \varnothing$ such that (i) $f$ is L-integrable on $J \cap E$, (ii) if $\left\{J_{k}=\left[a_{k}, b_{k}\right]\right\}$ are the intervals contiguous to $J \cap E$ with respect to $J$, then $\sum_{k=1}^{\infty} O\left(F, J_{k}\right)<\infty$, where $F$ is an indefinite integral of $f$.

Proof. By Property $1, F(x)$ is $\mathrm{ACG}^{*}$ on $[a, b]$ and $F^{\prime}(x)=f(x)$ a.e. Since $F$ is $\mathrm{ACG}^{*}$, there exists a closed interval $J$ with $J^{0} \cap E \neq \varnothing$ such that $F$ is $\mathrm{AC}^{*}$ on $J^{0} \cap E$ [5, p. 233]. Therefore $F$ is $\mathrm{AC}^{*}$ on its closure $\overline{J^{0} \cap E}$ by the continuity of $F$. An end point of $J$ may be $J \cap E$ without being in $\overline{J^{0} \cap E}$; but if this is so, then the end point is an isolated point of $J \cap E$. Hence $F$ is $\mathrm{AC}^{*}$ on $J \cap E$, and is VB* [5, p. 228] on $J \cap E$. It follows from the definition of $\mathrm{VB}^{*}$ that $\Sigma_{k} O\left(F, J_{k}\right)<\infty$.

We denote by $G(x)$ the function which coincides with $F(x)$ on $J \cap E$ and is linear on each $J_{k}$. Then the function $G$ is AC* on $J$ and hence $G^{\prime}$ is L-integrable on $J$. Since $G^{\prime}(x)=F^{\prime}(x)=f(x)$ at almost all points of $J \cap E, f$ is L-integrable on $J \cap E$.

REMARK 1. By the definition of the $D^{*}$-integral [5, p. 241], we see that this proof is valid for the $D^{*}$-integral. Hence Theorem 1 is true for the $D^{*}$-integral.

REMARK 2. If we take a closed interval as $E$ in Theorem 1 , then we have the following proposition. If $f$ is $\mathrm{RC}$-integrable on $[a, b]$ then there exists a closed subinterval $J$ on which $f$ is L-integrable.

THEOREM 2. The RC-integral is equivalent to the $D^{*}$-integral.

Proof. Throughout the proof, $I, J$ denote closed intervals.

Let $f$ be an RC-integrable function on $I_{0}=[a, b]$. Then, by Property 1 , its indefinite integral $F$ is $\mathrm{ACG}^{*}$ on $I_{0}$ and $F^{\prime}(x)=f(x)$ a.e. Hence, by the definition of the $D^{*}$-integral, $f$ is $D^{*}$-integrable and

$$
\left(D^{*}\right) \int_{a}^{b} f=(R C) \int_{a}^{b} f
$$


To prove the converse, let $f$ be $\mathrm{D}^{*}$-integrable on $I_{0}$ and $I$ be a subinterval of $I_{0}$. Then $f$ is allowed to take infinite values at the points of the set with measure zero. However these infinite values may be replaced by zero without altering the integral. Hence we can assume that $f$ is finite at every point.

If $f$ is RC-integrable on $I$ and $(R C) \int_{I} f=\left(D^{*}\right) \int_{I} f$, then we call the interval $I$ regular. By Remark 2 , there exists an interval $J$ on which $f$ is L-integrable. Since the RC-integral includes the L-integral (Property 2), $J$ is a regular interval. If $x_{0}$ is an interior point of some regular interval, then $x_{0}$ is called a regular point. Otherwise we call the point irregular.

(i) First we shall show that if the interval $I$ has no irregular points in its interior then any interval $J \subset I^{0}$ is regular.

Suppose that there exists an interval $J$ which is not regular. If we divide $J$ into two intervals by its middle point then at least one of these intervals is not regular since the integrals $\mathrm{RC}$ and $\mathrm{D}^{*}$ both have the additivity of the intervals. Continuing this process indefinitely, we have a sequence of intervals $\left\{J_{k}\right\}$ which are not regular. Let $x_{0}$ be the common point of the $J_{k}$. Then $x_{0}$ is an irregular point contained in $I^{0}$, which contradicts the hypothesis.

(ii) Next we shall show that if any interval $J \subset I^{0}$ is regular, then $I$ itself is regular.

We put $I=[\alpha, \beta]$ and $J=[c, d]$ where $\alpha<c<d<\beta$. Since $J$ is regular, we have, by definition, $(R C) \int_{c}^{d} f=\left(D^{*}\right) \int_{c}^{d} f$. It follows from the Cauchy property of $D^{*}$-integral $[5$, p. 247] that

$$
\lim _{\substack{c \rightarrow \alpha \\ d \rightarrow \beta}}(R C) \int_{c}^{d} f=\left(D^{*}\right) \int_{\alpha}^{\beta} f .
$$

Hence, by Property 3, $f$ is RC-integrable on $I$, and $(R C) \int_{\alpha}^{\beta} f=\left(D^{*}\right) \int_{\alpha}^{\beta} f$.

(iii) We have to show that $I_{0}$ is a regular interval. Suppose that $I_{0}$ is not regular. Then, by (i) and (ii), $I_{0}$ contains at least one irregular point. Let $E$ be the set of all irregular points. Then $E$ is closed. It follows from Remark 1 that there exists an interval $J$ with $J^{0} \cap E \neq \varnothing$ such that $f$ is L-integrable on $J \cap E$ and if $\left\{J_{k}=\right.$ $\left.\left[a_{k}, b_{k}\right]\right\}$ is the set of contiguous intervals of $J \cap E$ with respect to $J$, then $\Sigma_{k} O\left(D^{*}, f, J_{k}\right)<\infty$ where $O\left(D^{*}, f, J_{k}\right)$ means the oscillation of indefinite $\mathrm{D}^{*}$-integral on $J_{k}$. Hence, by the Harnack property of the $D^{*}$-integral [5, p. 257], we have

$$
\left(D^{*}\right) \int_{J} f=(L) \int_{J \cap E} f+\sum_{k=1}^{\infty}\left(D^{*}\right) \int_{J_{k}} f
$$

Since $J_{k}^{0} \cap E \neq \varnothing$, it follows from (i) and (ii) that each $I_{k}$ is regular and that

$$
\begin{gathered}
\left(D^{*}\right) \int_{J_{k}} f=(R C) \int_{J_{k}} f, \\
\sum_{k=1}^{\infty} O\left(D^{*}, f, J_{k}\right)=\sum_{k=1}^{\infty} O\left(F_{k}, J_{k}\right)<\infty,
\end{gathered}
$$


where $F_{k}$ is an indefinite $\mathrm{RC}$-integral on $J_{k}$. Hence we have from Property 4 that

$$
\begin{aligned}
\left(D^{*}\right) \int_{J} f & =(L) \int_{J \cap E} f+\sum_{k=1}^{\infty}\left(D^{*}\right) \int_{J_{k}} f \\
& =(L) \int_{J \cap E} f+\sum_{k=1}^{\infty}(R C) \int_{J_{k}} f=(R C) \int_{J} f .
\end{aligned}
$$

Therefore $J$ is regular. But this contradicts the relation $J^{0} \cap E \neq \varnothing$. Hence $I_{0}$ must be regular, which completes the proof.

\section{REFERENCES}

1. R. O. Davies and Z. Schuss, A proof that Henstock's integral includes Lebesgue's, J. London Math. Soc. (2) 2 (1970), 561-562.

2. R. Henstock, $A$ new descriptive definition of the Ward integral, J. London Math. Soc. 35 (1960), 43-48.

3. Theory of integration, Butterworths, London, 1963.

4. __ A Riemann-type integral of Lebesgue power, Canad. J. Math. 20 (1968), 79-87.

5. S. Saks, Theory of the integral, 2nd rev. ed., Dover, New York, 1964.

6. A. J. Ward, The Perron-Stieltjes integral, Math. Z. 41 (1936), 578-604.

Department of MAThematics, Ibaraki University, Mrto, Ibaraki, JaPan 